\title{
Bounds for the approximation of Poisson-binomial distribution by Poisson distribution
}

\author{
Tran Loc Hung* and Vu Thi Thao
}

"Correspondence:

tlhungvn@gmail.com

Faculty of Basic Science, University

of Finance \& Marketing (UFM),

306 Nguyen Trong Tuyen St., Tan

Binh Dist., Ho Chi Minh City,

Vietnam

\section{Springer}

\begin{abstract}
Let $\left(X_{n k}, k=1,2, \ldots, n ; n=1,2, \ldots\right)$ be a row-wise triangular array of independent Bernoulli random variables with success probabilities

$P\left(X_{n k}=1\right)=1-P\left(X_{n k}=0\right)=p_{n k} \in[0,1], k=1,2, \ldots, n ; n=1,2, \ldots$ For every $n=1,2, \ldots$, the random variables $S_{n}=\sum_{k=1}^{n} X_{n k}$ have probability distributions with complicated structure and therefore they are used to being approximated by Poisson distribution. Well-known Le Cam's inequality is established for providing information on the quality of a Poisson approximation. The main aim of this paper is to re-establish the Le Cam-type inequalities via a linear operator. The operator method used in this paper is quite elementary and it also could be applied for the probability distributions of random sums $S_{N_{n}}=\sum_{k=1}^{N_{n}} X_{n k}$ in the Poisson approximation, where $N_{n}, n=1,2, \ldots$, are positive integer-valued random variables, independent of all $X_{n k}, k=1,2, \ldots, n$; $n=1,2, \ldots$

MSC: $60 \mathrm{~F} 05 ; 60 \mathrm{G} 50 ; 41 \mathrm{~A} 36$

Keywords: Poisson distribution; Poisson-binomial random variable; Le Cam's inequality; linear operator; random sum; convergence in distribution; convergence in probability
\end{abstract}

\section{Introduction}

Throughout this paper, let $\left(X_{n k}, k=1,2, \ldots, n ; n=1,2, \ldots\right)$ be a row-wise triangular array of independent Bernoulli random variables with success probabilities $P\left(X_{n k}=1\right)=$ $1-P\left(X_{n k}=0\right)=p_{n k} \in[0,1], k=1,2, \ldots, n ; n=1,2, \ldots$ The random variables $S_{n}=\sum_{k=1}^{n} X_{n k}$, $n=1,2, \ldots$, are often called the Poisson-binomial random variables. And it is easily seen that the mean, variance, and characteristic function of $S_{n}, n=1,2, \ldots$, are $E\left(S_{n}\right)=$ $\sum_{k=1}^{n} p_{n k}, D\left(S_{n}\right)=\sum_{k=1}^{n} p_{n k}\left(1-p_{n k}\right)$, and $f_{S_{n}}(t)=E\left(e^{i t S_{n}}\right)=\prod_{k=1}^{n}\left(1-p_{n k}+p_{n k} e^{i t}\right)$, respectively.

The probability distributions of $S_{n}, n=1,2, \ldots$, have many applications in various areas of mathematics and statistics such as reliability, survival analysis, survey sampling, econometrics, and so on (the reader is referred to [1,2] and [3] for full development). However, since the probability distributions of $S_{n}, n \geq 1$, have the complicated structure (see, for instance, [3]), they are used to being approximated by the distribution of Poisson random variables $Z_{\lambda_{n}}$ with a positive parameter $\lambda_{n}=E\left(S_{n}\right)=\sum_{k=1}^{n} p_{n k}$. More specifically, assume 
that

$$
\lim _{n \rightarrow \infty} \lambda_{n}=\lambda \quad(0<\lambda<+\infty)
$$

then

$$
S_{n} \stackrel{d}{\rightarrow} Z_{\lambda}, \quad \text { as } n \rightarrow \infty
$$

where, and from now on, the notation $\stackrel{d}{\rightarrow}$ means the convergence in distribution (see, for instance, [4]). Moreover, remarkable Le Cam's inequality for the Poisson-binomial distribution [5] is widely considered in literature as follows:

$$
\sum_{k=0}^{\infty}\left|P\left(S_{n}=k\right)-P\left(Z_{\lambda_{n}}=k\right)\right| \leq 2 \sum_{k=1}^{n} p_{n k}^{2}
$$

(we refer the reader to the results of Le Cam [5], Barbour, Holst, and Janson [6], Steele [7], Chen [8], Chen and Liu [1], Neammanee [9], and Ross [10] for more details).

It should be noted that in [6,7], and [9] various powerful tools (such as the method of matrix analysis, the semi-group method, the coupling method, and the Chen-Stein method) for providing Le Cam's inequality have been demonstrated. The main objective of this paper is to obtain the bounds for well-known Le Cam's inequality in (3) using the operator method, introduced by Renyi [4]. In the third section, we use the operator method from [4] to establish the bounds for the approximation of Poisson-binomial distribution by Poisson distribution. The operator method in this paper is quite elementary and it also could be applied for random sums $S_{N_{n}}=\sum_{k=1}^{N_{n}} X_{n k}, S_{0}=0$, where $N_{n}, n=1,2, \ldots$ are positive integer-valued random variables, independent of all $X_{n k}, k=1,2, \ldots, n ; n=1,2, \ldots$. This will be taken up in the last section. We refer the reader to the works of Trotter [11], Renyi [4], and Hung [12] for a deeper discussion of this operator method. Based on the operator method, the received results of this paper are analogues of Le Cam's inequality in classical literature (we refer the reader to Steele [7], Le Cam [5], Chen [8], Neammanee [9], and Wang [3] for a complete treatment of the problem).

\section{Preliminaries}

In the sequel we will need the operator method, which has been used for a long time in various studies of classical limit theorems for sums of independent random variables (see Trotter [11], Renyi [4], and Hung [12] for the complete bibliography).

We recall some definitions and notations. We denote by $K$ the set of all real-valued bounded functions $f(x)$, defined on the set of non-negative integers $Z_{+}=\{0,1,2, \ldots\}$. The norm of a function $f \in K$ is defined by $\|f\|=\sup _{x \in Z_{+}}|f(x)|$.

Definition 2.1 We define a linear operator associated with a positive discrete random variable $X, A_{X}: K \rightarrow K$, by setting

$$
\left(A_{X} f\right)(x):=E(f(X+x))=\sum_{k=0}^{\infty} f(x+k) P(X=k), \quad \forall f \in K, x \in Z_{+} .
$$


It is to be noticed that the linear operator defined in (4) is actually a discrete form of Trotter's operator (we refer the readers to Trotter [11], Renyi [4], and Hung [12] for a more general and detailed discussion of this operator method).

We will need some properties of the operator in (4) in the sequel. Let $A_{X}, A_{Y}$ be operators associated with two discrete random variables $X$ and $Y$ for $f, g \in K$. Suppose that $\alpha$ and $\beta$ are two real numbers, then we easily get the following linear property of the operator in (4):

$$
A_{X}(\alpha f+\beta g)=\alpha A_{X}(f)+\beta A_{X}(g) .
$$

We define the operator $\left(A_{X}+A_{Y}\right)$ by $\left(A_{X}+A_{Y}\right) f=A_{X} f+A_{Y} f, \forall f \in K$, and the product of two operators $A_{X}$ and $A_{Y}$ is $\left(A_{X} A_{Y}\right) f=A_{X}\left(A_{Y} f\right), \forall f \in K$.

It is obvious that

1. $\left\|A_{X} f\right\| \leq\|f\|$ for all $f \in K$.

2. $\left\|A_{X} f+A_{Y} f\right\| \leq\left\|A_{X} f\right\|+\left\|A_{Y} f\right\|$ for all $f \in K$.

3. Suppose that $A_{X}$ and $A_{Y}$ are operators associated with two independent random variables $X, Y$ and $f \in K$. Then $A_{X} A_{Y} f=A_{Y} A_{X} f=A_{X+Y} f$.

In fact, for all $f \in K$ and $x \in Z_{+}$,

$$
\begin{aligned}
A_{X} A_{Y} f(x) & =A_{X}\left(A_{Y} f(x)\right)=A_{X}\left(\sum_{k=0}^{\infty} f(x+k) P(Y=k)\right) \\
& =\sum_{r, k=0}^{\infty} f(x+k+r) P(Y=k) P(X=r) \\
& =\sum_{l=0}^{\infty} f(x+l) P(X+Y=l) \\
& =A_{X+Y} f(x)
\end{aligned}
$$

by an argument analogous to that used for the proof of $A_{Y} A_{X} f=A_{X+Y} f$.

4. Suppose that $A_{X_{1}}, A_{X_{2}}, \ldots, A_{X_{n}}$ are the operators associated with the independent random variables $X_{1}, X_{2}, \ldots, X_{n}$. Then $A_{S_{n}}=A_{X_{1}} A_{X_{2}} \cdots A_{X_{n}}$ is the operator associated with the partial sum $S_{n}=X_{1}+X_{2}+\cdots+X_{n}$.

5. Suppose that $A_{X_{1}}, A_{X_{2}}, \ldots, A_{X_{n}}$ and $A_{Y_{1}}, A_{Y_{2}}, \ldots, A_{Y_{n}}$ are operators associated with independent random variables $X_{1}, X_{2}, \ldots, X_{n}$ and $Y_{1}, Y_{2}, \ldots, Y_{n}$. Moreover, assume that all $X_{i}$ and $Y_{j}$ are independent for $i, j=1,2, \ldots, n$. Then, for every $f \in K$,

$$
\left\|A_{\sum_{k=1}^{n} X_{k}} f-A_{\sum_{k=1}^{n} Y_{k}} f\right\| \leq \sum_{k=1}^{n}\left\|A_{X_{k}} f-A_{Y_{k}} f\right\| .
$$

Clearly,

$$
A_{X_{1}} A_{X_{2}} \cdots A_{X_{n}}-A_{Y_{1}} A_{Y_{2}} \cdots A_{Y_{n}}=\sum_{k=1}^{n} A_{X_{1}} A_{X_{2}} \cdots A_{X_{k-1}}\left(A_{X_{k}}-A_{Y_{k}}\right) A_{Y_{k+1}} \cdots A_{Y_{n}} .
$$


It deduces that

$$
\begin{aligned}
\left\|A_{\sum_{k=1}^{n} X_{k}} f-A_{\sum_{k=1}^{n} Y_{k}} f\right\| & \leq \sum_{k=1}^{n}\left\|A_{X_{1}} \cdots A_{X_{k-1}}\left(A_{X_{k}}-A_{Y_{k}}\right) A_{Y_{k+1}} \cdots A_{Y_{n}} f\right\| \\
& \leq \sum_{k=1}^{n}\left\|A_{Y_{k+1}} \cdots A_{Y_{n}}\left(A_{X_{k}}-A_{Y_{k}}\right) f\right\| \\
& \leq \sum_{k=1}^{n}\left\|A_{X_{k}} f-A_{Y_{k}} f\right\| .
\end{aligned}
$$

6. It is to be noticed that $\left\|A_{X}^{n} f-A_{Y}^{n} f\right\| \leq n\left\|A_{X} f-A_{Y} f\right\|$.

7. Suppose that $X_{1}, \ldots, X_{n}$ and $Y_{1}, \ldots, Y_{n}$ are independent random variables (in each group), and let $\left\{N_{n}, n=1,2, \ldots\right\}$ be a sequence of positive integer-valued random variables independent of all $X_{k}$ and $Y_{k}, k=1,2, \ldots$ Then, for every $f \in K$,

$$
\left\|A_{\sum_{k=1}^{N_{n}} X_{k}} f-A_{\sum_{k=1}^{N_{n}} Y_{k}} f\right\| \leq \sum_{n=1}^{\infty} P\left(N_{n}=n\right) \sum_{k=1}^{n}\left\|A_{X_{k}} f-A_{Y_{k}} f\right\| .
$$

Lemma 2.1 The equation $A_{X} f(x)=A_{Y} f(x)$ for $f \in K, x \in Z_{+}$, provided that $X$ and $Y$ are identically distributed random variables.

Let $A_{X_{1}}, A_{X_{2}}, \ldots, A_{X_{n}}, \ldots$ be a sequence of operators associated with the independent discrete random variables $X_{1}, X_{2}, \ldots, X_{n}, \ldots$, and $A_{X}$ be the operator associated with the discrete random variable $X$. The following lemma states one of the most important properties of the operator $A_{X}$.

Lemma 2.2 A sufficient condition for a sequence of random variables $X_{1}, X_{2}, \ldots, X_{n} \ldots$ converging in distribution to a random variable $X$ is that

$$
\lim _{n \rightarrow \infty}\left\|A_{X_{n}} f-A_{X} f\right\|=0 \quad \text { for all } f \in K
$$

Proof Since $\lim _{n \rightarrow \infty}\left\|A_{X_{n}} f-A_{X} f\right\|=0$, for all $f \in K$, we get

$$
\lim _{n \rightarrow \infty}\left|\sum_{k=0}^{\infty} f(x+k)\left(P\left(X_{n}=k\right)-P(X=k)\right)\right|=0 \quad \text { for all } f \in K \text { and } x \in Z_{+} .
$$

If we choose

$$
f(x)= \begin{cases}1, & \text { if } 0 \leq x \leq t \\ 0, & \text { if } x>t\end{cases}
$$

Then

$$
\lim _{n \rightarrow \infty}\left|\sum_{k=0}^{t}\left(P\left(X_{n}=k\right)-P(X=k)\right)\right|=0 .
$$

It follows that $P\left(X_{n} \leq t\right)-P(X \leq t) \rightarrow 0$ as $\mathrm{n}$ tends to $+\infty$.

In other words, $X_{n} \stackrel{d}{\rightarrow} X$ as $n \rightarrow+\infty$. 


\section{A bound of Poisson-binomial approximation}

Let $A_{X_{n k}}, k=1, \ldots, n ; n=1,2, \ldots$ be the operators associated with the random variables $X_{n k}$, $k=1, \ldots, n ; n=1,2, \ldots$, and let $A_{Z_{p_{n k}}}, k=1, \ldots, n ; n=1,2, \ldots$, be the operators associated with the Poisson random variables with parameters $p_{n k}, k=1, \ldots, n ; n=1,2, \ldots$ On the assumption that $Z_{\lambda_{n}}$ is a Poisson random variable with a positive parameter $\lambda_{n}=\sum_{k=1}^{n} p_{n k}$, we can perform that $Z_{\lambda_{n}} \stackrel{d}{=} \sum_{k=1}^{n} Z_{p_{n k}}$, where $Z_{p_{n 1}}, Z_{p_{n 2}}, \ldots, Z_{p_{n n}}$ are independent Poisson random variables with positive parameters $p_{n 1}, p_{n 2}, \ldots, p_{n n}$, and the notation $\stackrel{d}{=}$ denotes coincidence of distributions. We will now state an analogue of Le Cam's inequality [5] via the linear operator in (4) as follows.

Theorem 3.1 Let $\left(X_{n k}, 1 \leq k \leq n ; n=1,2, \ldots\right)$ be a row-wise triangular array of independent, Bernoulli random variables with success probabilities $P\left(X_{n k}=1\right)=1-P\left(X_{n k}=0\right)=$ $p_{n k}, p_{n k} \in[0,1], k=1,2, \ldots, n ; n=1,2, \ldots$. Let us write $S_{n}=\sum_{k=1}^{n} X_{n k}$ and $\lambda_{n}=\sum_{k=1}^{n} p_{n k}$. We denote by $Z_{\lambda_{n}}$ the Poisson random variable with the parameter $\lambda_{n}$. Then, for all real-valued bounded functions $f \in K$, we have

$$
\left\|A_{S_{n}} f-A_{Z_{\lambda_{n}}} f\right\| \leq 2\|f\| \sum_{k=1}^{n} p_{n k}^{2}
$$

Proof Applying the inequality in (5), we have

$$
\left\|A_{S_{n}} f-A_{Z_{\lambda n}} f\right\| \leq \sum_{k=1}^{n}\left\|A_{X_{n k}} f-A_{Z_{p_{n k}}} f\right\| .
$$

Moreover, for all $f \in K$ and for all $x \in Z_{+}$, we conclude that

$$
\begin{aligned}
A_{X_{n k}} f(x)-A_{Z_{p_{n k}}} f(x)= & \sum_{r=0}^{\infty} f(x+r)\left(P\left(X_{n k}=r\right)-P\left(Z_{p_{n k}}=r\right)\right) \\
= & \sum_{r=0}^{\infty} f(x+r)\left(P\left(X_{n k}=r\right)-\frac{e^{-p_{n k}} p_{n k}^{r}}{r !}\right) \\
= & f(x)\left(1-p_{n k}-e^{-p_{n k}}\right)+f(x+1)\left(p_{n k}-p_{n k} e^{-p_{n k}}\right) \\
& -\sum_{r=2}^{\infty} f(x+r) \frac{e^{-p_{n k}} p_{n k}^{r}}{r !} .
\end{aligned}
$$

Since $\sum_{r=2}^{\infty} \frac{e^{-p_{n k}} p_{n k}^{r}}{r !}=1-e^{-p_{n k}}-p_{n k} e^{-p_{n k}}$, for all $f \in K$ and $x \in Z_{+}$, it may be concluded that

$$
\begin{aligned}
\left|A_{X_{n k}}(f)-A_{Z_{p_{n k}}}(f)\right|= & \mid f(x)\left(1-p_{n k}-e^{-p_{n k}}\right)+f(x+1)\left(p_{n k}-p_{n k} e^{-p_{n k}}\right) \\
& -\sum_{r=2}^{\infty} f(x+r) \frac{e^{-p_{n k}} p_{n k}^{r}}{r !} \mid \\
\leq & \left|f(x)\left(1-p_{n k}-e^{-p_{n k}}\right)\right|+\left|f(x+1)\left(p_{n k}-p_{n k} e^{-p_{n k}}\right)\right| \\
& +\left|\sum_{r=2}^{\infty} f(x+r) \frac{e^{-p_{n k}} p_{n k}^{r}}{r !}\right|
\end{aligned}
$$




$$
\begin{aligned}
& \leq \sup _{x \in Z^{+}}|f(x)|\left(e^{-p_{n k}}-1+p_{n k}+p_{n k}-p_{n k} e^{-p_{n k}}+1-e^{-p_{n k}}-p_{n k} e^{-p_{n k}}\right) \\
& \leq 2\|f\| p_{n k}\left(1-e^{-p_{n k}}\right) \leq 2\|f\| p_{n k}^{2} .
\end{aligned}
$$

Therefore, applying (5), we can assert that

$$
\left\|A_{S_{n}}(f)-A_{Z_{\lambda_{n}}}(f)\right\| \leq 2\|f\| \sum_{k=1}^{n} p_{n k}^{2} .
$$

This completes the proof.

Remark 3.1 According to Theorem 3.1 and assumption (1), using the definition of the norm of the operator $A$, we get following inequality:

$$
\left\|A_{S_{n}}-A_{Z_{\lambda}}\right\| \leq 2\left(\sum_{k=1}^{n} p_{n k}^{2}\right)
$$

The following corollaries are immediate consequences from Theorem 3.1.

Corollary 3.1 Under the stated assumptions of Theorem 3.1 , for all $k=0,1,2, \ldots$,

$$
\left|P\left(S_{n}=k\right)-P\left(Z_{\lambda_{n}}=k\right)\right| \leq 2 \sum_{j=1}^{n} p_{n j}^{2} .
$$

Proof Choose the particular function $f(x), x \in Z_{+}$, such that

$$
f(x+m)= \begin{cases}1 & \text { if } m=k \\ 0 & \text { if } m \neq k\end{cases}
$$

Set $y=x+m$. Since $x, m \in Z^{+}$, it follows that $y \in Z_{+}$. Then we have

$$
\|f\|=\sup _{x}|f(x)|=\sup _{y}|f(y)|=1
$$

Thus, according to Theorem 3.1, we conclude that

$$
\left\|A_{S_{n}}(f)-A_{Z_{\lambda_{n}}}(f)\right\| \leq 2 \sum_{j=1}^{n} p_{n j}^{2} .
$$

On the other hand, by choosing the function $f(x)$ as above, we have

$$
\begin{aligned}
\left\|A_{S_{n}} f-A_{Z_{\lambda_{n}}} f\right\| & =\sup _{x}\left|A_{S_{n}} f(x)-A_{Z_{\lambda_{n}}} f(x)\right| \\
& =\sup _{x}\left|\sum_{m=0}^{\infty} f(x+m)\left[P\left(S_{n}=m\right)-P\left(Z_{\lambda_{n}}=m\right)\right]\right| \\
& =\sup _{x} \mid f(x)\left[P\left(S_{n}=0\right)-P\left(Z_{\lambda_{n}}=0\right)\right]+\cdots
\end{aligned}
$$




$$
\begin{aligned}
& +f(x+k)\left[P\left(S_{n}=k\right)-P\left(Z_{\lambda_{n}}=k\right)+\cdots\right] \mid \\
= & \left|P\left(S_{n}=k\right)-P\left(Z_{\lambda_{n}}=k\right)\right| .
\end{aligned}
$$

Applying (8) we can assert that

$$
\left|P\left(S_{n}=k\right)-P\left(Z_{\lambda_{n}}=k\right)\right| \leq 2 \sum_{j=1}^{n} p_{n j}^{2}
$$

The proof is complete.

Corollary 3.2 Let condition (1) hold. Under the hypotheses of Theorem 3.1, if moreover

$$
\lim _{n \rightarrow \infty} \max _{1 \leq k \leq n} p_{n k}=0
$$

then the distribution of $S_{n}$ converges to the Poisson distribution with mean $\lambda$, i.e., $S_{n} \stackrel{d}{\rightarrow} Z_{\lambda}$ as $n \rightarrow \infty$.

Proof The proof is based on the following observation:

$$
\sum_{k=1}^{n} p_{n k}^{2} \leq \max _{1 \leq k \leq n} p_{n k} \times \sum_{k=1}^{n} p_{n k}
$$

According to the inequality in (6) for all $f \in K$ and (9), we conclude that

$$
\lim _{n \rightarrow \infty}\left\|A_{S_{n}}(f)-A_{Z_{\lambda_{n}}}(f)\right\|=0
$$

As an argument analogous to the one used for the proof of Corollary 3.1, on account of Lemma 2.2, we get

$$
\lim _{n \rightarrow \infty}\left[P\left(S_{n}=k\right)-P\left(Z_{\lambda_{n}}=k\right)\right]=0 .
$$

Then, on account of (1), we have

$$
\lim _{n \rightarrow \infty} P\left(S_{n}=k\right)=\lim _{n \rightarrow \infty} \frac{e^{-\lambda_{n}}\left(\lambda_{n}\right)^{k}}{k !}=\frac{e^{-\lambda} \lambda^{k}}{k !}, \quad k=0,1,2 \ldots
$$

Thus, the proof is straightforward.

\section{A bound of random Poisson-binomial approximation}

Throughout this section, we begin with assuming that $N_{n}, n=1,2, \ldots$, are positive integervalued random variables independent of all $X_{n k}, k=1,2, \ldots, n ; n=1,2, \ldots$, which are supposed to obey the relation

$$
N_{n} \stackrel{P}{\rightarrow}+\infty \quad \text { as } n \rightarrow+\infty
$$

Here and subsequently, $\stackrel{P}{\rightarrow}$ denotes the convergence in probability. For every $n=1,2, \ldots$, we denote by $S_{N_{n}}$ the random sums $S_{N_{n}}=\sum_{k=1}^{N_{n}} X_{n k}\left(S_{0}=0\right.$ by convention). Therefore, the 
random sums $S_{N_{n}}$ could be said to be the random Poisson-binomial random variables. In this section, we establish Le Cam-type inequalities related to the Poisson approximation for distributions of random Poisson-binomial variables. It is to be noticed that many various results concerning the random summations have already been included in the textbooks of probability theory; see, e.g., $[4,13,14])$.

Let $A_{X_{n 1}}, A_{X_{n 2}}, \ldots, A_{X_{n N_{n}}}$ be operators associated with the independent triangular array of random variables $X_{n 1}, X_{n 2}, \ldots, X_{n N_{n}}$, and let $A_{Z_{p_{n 1}}}, A_{Z_{p_{n 2}}}, \ldots, A_{Z_{p_{n N}}}$ be operators associated with the independent Poisson distributed random variables with positive parameters $p_{n 1}, p_{n 2}, \ldots, p_{n N_{n}}$. According to the properties of the linear operator in (4), we have $A_{S_{N_{n}}}=A_{X_{n 1}} A_{X_{n 2}} \cdots A_{X_{n N_{n}}}$ and $A_{Z_{\lambda_{n}}}=A_{Z_{p_{n 1}}} A_{Z_{p_{n 2}}} \cdots A_{Z_{p_{n N}}}$ are the respective operators associated with the random sums $S_{N_{n}}=\sum_{k=1}^{N_{n}} X_{n k}$ and $Z_{\lambda_{n}}=\sum_{k=1}^{N_{n}} Z_{p_{n k}}$.

Theorem 4.1 Let $\left(X_{n k}, k=1,2, \ldots, n ; n=1,2, \ldots\right)$ be a row-wise triangular array of independent, non-identically distributed Bernoulli random variables with success probabilities $P\left(X_{n k}=1\right)=1-P\left(X_{n k}=0\right)=p_{n k}, p_{n k} \in[0,1], k=1,2, \ldots, n ; n=1,2, \ldots$ Moreover, we suppose that $N_{n}, n=1,2, \ldots$ are independent positive integer-valued random variables, independent of all $X_{n k}, k=1,2, \ldots, n ; n=1,2, \ldots$. Then, for all real-valued bounded functions $f \in K$ and for all $x \in Z_{+}$, we have

$$
\left\|A_{S_{N_{n}}}(f)-A_{Z_{\lambda_{N}}}(f)\right\| \leq 2\|f\| E\left(\sum_{k=1}^{N_{n}} p_{n k}^{2}\right) .
$$

Proof According to the assumptions on the random variables $N_{n}, X_{n k}, Z_{\lambda_{n}}, k=1,2, \ldots, n$; $n=1,2, \ldots$, we can write

$$
A_{S_{N_{n}}} f(x)=\sum_{m=1}^{\infty} P\left(N_{n}=m\right) \sum_{k=0}^{\infty} f(x+k) P\left(S_{m}=k\right),
$$

and

$$
A_{Z_{\lambda_{N}}} f(x)=\sum_{m=1}^{\infty} P\left(N_{n}=m\right) \sum_{k=0}^{\infty} f(x+k) \frac{e^{-\lambda_{m}} \lambda_{m}^{k}}{k !} .
$$

Therefore, by an argument analogous to that used for the proof of Theorem 3.1, for all real-valued function $f \in K, x \in Z_{+}$, we have

$$
\begin{aligned}
\left\|A_{S_{N_{n}}}(f)-A_{Z_{\lambda_{N}}}(f)\right\| & =\left\|\sum_{m=1}^{\infty} P\left(N_{n}=m\right)\left(A_{X_{n 1}} \cdots A_{X_{n m}}(f)-A_{Z_{p_{n 1}}} \cdots A_{Z_{p_{n m}}}(f)\right)\right\| \\
& \leq \sum_{m=1}^{\infty} P\left(N_{n}=m\right)\left\|A_{X_{n 1}} \cdots A_{X_{n m}}(f)-A_{Z_{p_{n 1}}} \cdots A_{Z_{p_{n m}}}(f)\right\| \\
& \leq \sum_{m=1}^{\infty} P\left(N_{n}=m\right) \sum_{k=1}^{m} 2\|f\| p_{n k}^{2} \\
& \leq 2\|f\| E\left(\sum_{k=1}^{N_{n}} p_{n k}^{2}\right) .
\end{aligned}
$$

The proof is complete. 
Note that the following remarks are immediate consequences from Theorem 4.1.

Remark 4.1 According to Theorem 4.1 and assumption (1), using the definition of the norm of the operator $A$, we conclude that

$$
\left\|A_{S_{N_{n}}}-A_{Z_{\lambda}}\right\| \leq 2 E\left(\sum_{k=1}^{N_{n}} p_{n k}^{2}\right) .
$$

Remark 4.2 By an argument analogous to that used for the proof of Corollary 3.1, under the stated assumptions of Theorem 4.1, for all $k=0,1,2, \ldots$, we have

$$
\left|P\left(S_{N_{n}}=k\right)-P\left(Z_{\lambda_{N_{n}}}=k\right)\right| \leq 2 E\left(\sum_{k=1}^{N_{n}} p_{n k}^{2}\right) .
$$

When the success probability is identical, $p_{n k}=p_{n} \in[0,1], k=1,2, \ldots, n$; for $n=1,2, \ldots$, we obtain the following remark.

Remark 4.3 Suppose that the $N_{n}, n=1,2, \ldots$ are positive integer-valued random variables independent of all independent identically distributed random variables $X_{n k}$, and assume that $P\left(X_{n k}=1\right)=1-P\left(X_{n k}=0\right)=p_{n} \in[0,1], k=1,2, \ldots, N_{n} ; n=1,2, \ldots$ Then, for all $k=$ $0,1,2, \ldots$, we get the following inequality:

$$
\left|P\left(S_{N_{n}}=k\right)-P\left(Z_{\lambda_{N_{n}}}=k\right)\right| \leq 2 E\left(N_{n}\right) p_{n}^{2} .
$$

It is worth noticing that when the positive integer-valued random variables $N_{n}, n=$ $1,2, \ldots$ take on the value $n$ with probability one, i.e., $P\left(N_{n}=n\right)=1$, the results concerning the probability distributions of the random sums $S_{N_{n}}$ in the Poisson approximation in this section return to the ones in Section 3.

We conclude this paper with the following comments. The linear operator in this paper introduced by Renyi [4] essentially is a discrete form of Trotter's operator [11] which has been used in the theory of limit theorems. The proofs of theorems in this paper by the operator method are very elementary and elegant. The received results in this article allow us to think about a new approach method to the Poisson approximation problems for the distributions of the sums of the discrete independent random variables like Poissonbinomial, geometric, and negative binomial variables.

Competing interests

The authors declare that they have no completing interests.

Authors' contributions

All authors read and approved the final manuscript.

\section{Acknowledgements}

Dedicated to Professor Nguyen Duy Tien on the occasion of his 70th birthday.

The authors wish to express their gratitude to the referees for valuable remarks and comments improving the previous version of this paper. This work is supported by the Vietnam National Foundation For Science and Technology

Development (NAFOSTED, Vietnam), grant 101.01-2010.02. 


\section{References}

1. Chen, SX, Liu, JS: Statistical applications of the Poisson-binomial and conditional Bernoulli distributions. Stat. Sin. 7, 875-892 (1997)

2. Tejada, A, Dekker, AJ: The role of Poisson's binomial distribution in the analysis of TEM images. Ultramicroscopy 111 , 1553-1556 (2011)

3. Wang, YH: On the number of successes in independent trials. Stat. Sin. 3, 295-312 (1993)

4. Renyi, A: Probability Theory. Akad. Kiadó, Budapest (1970)

5. Le Cam, L: An approximation theorem for the Poisson binomial distribution. Pac. J. Math. 10(4), 1181-1197 (1960)

6. Barbour, AD, Holst, L, Janson, S: Poisson Approximation. Clarendon, Oxford (1992)

7. Steele, JM: Le Cam's inequality and Poisson approximations. Am. Math. Mon. 101(1), 48-54 (1994)

8. Chen, LHY: On the convergence of Poisson binomial to Poisson distribution. Ann. Probab. 2(1), 178-180 (1974)

9. Neammanee, K: A nonuniform bound for the approximation of Poisson binomial by Poisson distribution. Int. J. Math. Math. Sci. 2003(48), 3041-3046 (2003)

10. Ross, SM: Introduction to Probability Models, 9th edn. Elsevier, Amsterdam (2007)

11. Trotter, HF: An elementary proof of the central limit theorem. Arch. Math. 10, 226-234 (1959)

12. Hung, TL: On a probability metric based on Trotter operator. Vietnam J. Math. 35(1), 22-33. (2007)

13. Kruglov, VM, Korolev, VY: Limit Theorems for Random Sums. Moscow University Press, Moscow (1990) (in Russian)

14. Gnedenko, BV, Korolev, VY: Random Summation: Limit Theorems and Applications. CRC Press, Boca Raton (1996)

doi:10.1186/1029-242X-2013-30

Cite this article as: Hung and Thao: Bounds for the approximation of Poisson-binomial distribution by Poisson distribution. Journal of Inequalities and Applications 2013 2013:30.

\section{Submit your manuscript to a SpringerOpen ${ }^{\circ}$ journal and benefit from:}

- Convenient online submission

- Rigorous peer review

- Immediate publication on acceptance

- Open access: articles freely available online

- High visibility within the field

- Retaining the copyright to your article 